Pulsar Astronomy - 2000 and Beyond

ASP Conference Series, Vol. 202, 2000

M. Kramer, N. Wex, and R. Wielebinski, eds.

\title{
Pulsar spectra analysis
}

Olaf Maron, Jarostaw Kijak

J. Kepler Astronomical Center, Pedagogical University, Lubuska 2, PL-65-265 Zielona Góra, Poland

Michael Kramer, Richard Wielebinski

Max-Planck-Institut für Radioastronomie, Auf dem Hügel 69, D-53121 Bonn, Germany

\begin{abstract}
We have extended the database of flux density measurements of 280 pulsars made by Lorimer et al. (1995) to frequencies higher than $1.4 \mathrm{GHz}$ and lower than $300 \mathrm{MHz}$. Our analysis shows that the spectral behaviour of the majority of pulsars can be well described by a simple power law $\left(S \sim \nu^{\alpha}\right.$ where $\left.\bar{\alpha}=-1.8\right)$ though some pulsar spectra are described by a "broken power law" with a break at high frequencies $\nu_{b} \sim 1.8 \mathrm{GHz}$.
\end{abstract}

\section{Introduction}

We have collected pulsar observations and obtained spectra of 280 objects. As a basis for our database we have taken the low frequency measurements by Lorimer et al. (1995). For the frequencies above $1.4 \mathrm{GHz}$ we have gathered the flux measurements from Effelsberg Radiotelescope. We have also included the data published by Izvekova et al. (1981) at frequencies from 39 to 102.5 $\mathrm{MHz}$. Most of the pulsar spectra in the range from $300 \mathrm{MHz}$ to $43 \mathrm{GHz}$ can be well approximated by a simple power law. We have distinguished a group of 37 pulsars with a broken-type spectra. The analysis shows spectra flattening at high frequencies. The values measured by Izvekova et al. 1981 are shown by squares, those by Lorimer et al. (1995) by diamonds and those measured in Effelsberg by circles.

\section{Analysis}

Our spectral analysis shows that pulsar spectra may be divided into two groups: simple with power law $S \sim \nu^{\alpha}$ and broken-type consisting of two power laws. 233 pulsars have simple power law spectra with spectral index $\bar{\alpha}=-1.8$. There are 37 pulsars with broken-type spectra for which $\bar{\alpha}_{1}=-0.9$ and $\bar{\alpha}_{2}=-2.2$. The avarage break frequency is $\bar{\nu}_{b}=1.8 \mathrm{GHz}$. There are some pulsars which have a simple power law spectrum with spectral index $\bar{\alpha} \geq-0.9$ (see example in Figure 1). Most of them where observed only at frequencies lower than 1.4 GHz. These pulsars probably belong to the broken-type group. 

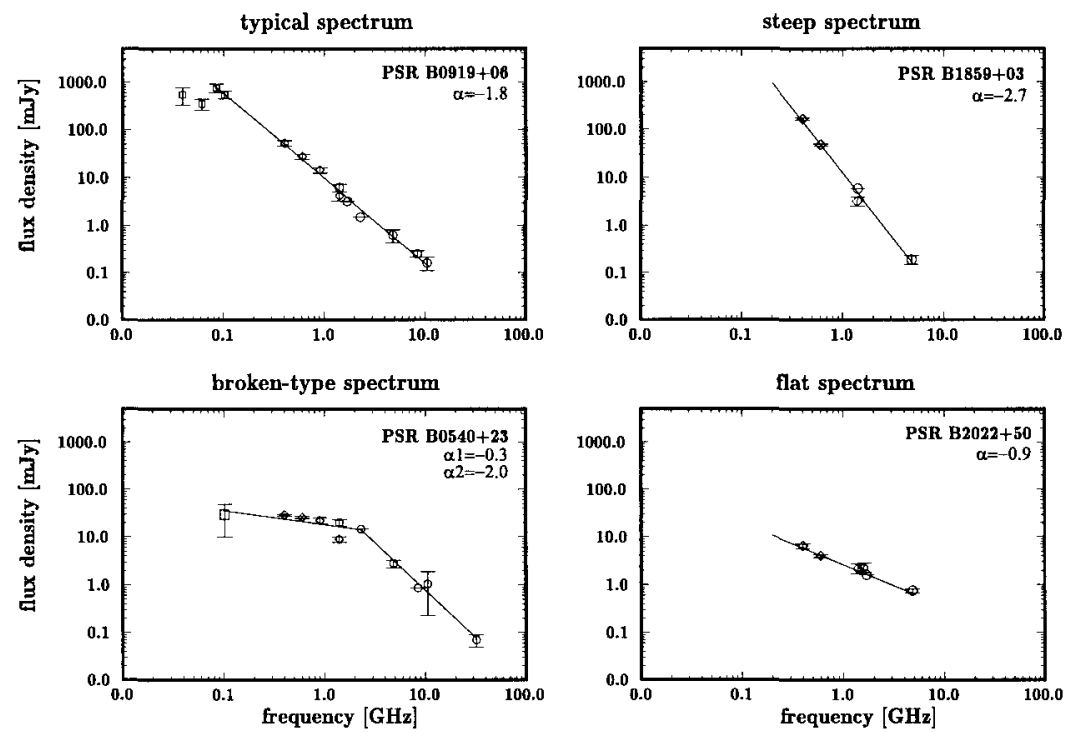

Figure 1. Examples of pulsar flux density spectrum, squares indicate measurements by Izvekova et al. 1981, diamonds - by Lorimer et al. 1995 and circles - measurements from Effelsberg Observatory

\section{Summary}

We presented the analysis of consistent sample of flux measurements. In general, pulsar spectra are steep with $\bar{\alpha}=-1.8 \pm 0.2$ (see also Malofeev in these proceedings). However, there is a group of pulsars whose spectra are broken-type. Some show flat spectra or flattening tendency at high frequencies (Maron et al., in preparation). Comparison of pulsar spectra analysis for normal and millisecond pulsars indicates that both groups have the same emission mechanism (cf. Kramer et al. 1998, Toscano et al. 1998).

Acknowledgments. This papers is partially supported by the Polish State Committee for Scientific Research Grant 2 P03D 01512.

\section{References}

Izvekova, V. A., Kuzmin, A. D., Mafofeev, V. M., Shitov, Y. P. 1981, Ap\&SS, 78,45

Kramer, M.,Xilouris, K. M., Lorimer, D. R., Doroshenko, O., Jessner, A., Wielebinski, R., Wolszczan, A., Camilo, F. 1998, ApJ, 501, 270

Lorimer, D. R., Yates, J. A., Lyne, A. G., Gould, D. M. 1995, MNRAS, 273, 411

Toscano, M., Bailes, M., Manchester, R. N., Sandhu, J. S. 1998, ApJ, 506, 863 